\title{
EDITORIAL
}

\section{Lessons learned: special precautions for performing emergency cerebrovascular procedures amid the COVID-19 pandemic}

\author{
Xiaoguang Tong, MD, ${ }^{1,2}$ and Yuanfan Yang, MD ${ }^{3,4}$ \\ ${ }^{1}$ Department of Neurosurgery, Tianjin Huanhu Hospital; ${ }^{2}$ Tianjin Neurosurgical Institute, Tianjin, China; and Departments of \\ ${ }^{3}$ Neurosurgery and ${ }^{4}$ Pathology, Duke University, Durham, North Carolina
}

$\mathrm{O}$ NE challenge of COVID-19 for neurosurgeons is that we must continue to provide critical neurosurgical care, even while there is substantial risk of exposing our provider teams while we perform emergency neurosurgical procedures. In this opinion piece, we describe the strategy for managing this problem at the Huanhu Hospital in Tianjin, China. We would like to note that after taking precautions in Tianjin based on our experience during the SARS outbreak, there is only 1 reported case of hospital staff infection (ICU doctor, mild case) locally. Among the fully equipped healthcare workers who were recruited to support Wuhan province (6200 nationally, 628 from Tianjin) since February 2020, there is no infection reported.

The current outbreak of the COVID-19 pandemic in China imparts great risk for infections in the context of providing emergency care, as unscreened stroke patients continue to rush into overwhelmed emergency rooms (ERs) needing emergency treatment and procedures. Our hospital is the municipal referral center for cerebrovascular disease, performing approximately 1000 emergency procedures for subarachnoid hemorrhage $(\mathrm{SAH})$ yearly and using interventional techniques to treat hundreds of patients with ischemic stroke. Most of our stroke patients are an elderly, vulnerable population who are already experiencing respiratory compromise, with the risk of respiratory failure potentially increased due to COVID-19 infection. In this context we have adopted the following strategies, which have proven to be effective during the pandemic.

\section{Preoperative Assessment in the ER}

We have updated our rapid screening and triage protocol in the ER. Because patients with SAH and other cerebrovascular diseases can present with fever and pulmonary infection, it is unrealistic to rapidly differentiate from COVID-19 co-infection based on symptoms alone. As community spread of COVID-19 has reached endemic proportions, we now regard all emergency cases as suspected COVID-19-positive cases until proven negative by testing. Personal protective equipment (PPE) was upgraded to an appropriate level for all personnel in the ER to include N95 masks, full gown, goggles, and face shield. Our observation has been that endotracheal intubation poses the highest risk for secondary transmission to healthcare workers and we recommend performing nasotracheal intubation whenever possible. Any emergency intubation must be done wearing 2 layers of face protection (goggles and face shield), and ideally under positive ventilation conditions.

\section{Emergency Surgery}

We found it useful in our regional trauma and stroke center in an endemic region to set up a "contamination zone" allowing patients to proceed directly from the ER to the OR isolated from the rest of the hospital. To meet the isolation requirements, our tertiary referral hospital assigned a separate imaging area serving the ER, with MRI (3T magnet), CT (CTA), and DSA for suspected cases. We also assigned an OR and catheter lab to serve this contamination zone with easy access to the imaging area. Suspected cases are moved to the OR directly from the ER, receiving proper ventilation while in the contamination zone to meet our current protocols for preventing nosocomial infection. Because airborne infection poses the highest risk of large-scale infection among healthcare workers, we have developed a separate ventilation system for the contamination zone.

For all stroke patients with suspected COVID-19 infection, we prioritize minimally invasive procedures over surgery whenever possible, in order to minimize the extent and duration of procedural exposure to hospital staff. In our experience, it is clear that coronavirus is present in the blood and secretions of infected patients, and all personnel 
involved in invasive procedures use specific precautions to avoid contacting body fluids and aerosols from suspected patients. This includes wearing full PPE with goggles and face shield to avoid fluid splashes, in particular.

Due to limitations of resources in the contamination zone, it is necessary to increase turnover and avoid overloading the contamination zone OR with extended cases. We also do the following: 1) Ensure that the air in the OR is disinfected between surgeries under a standard protocol approved by our infection control team. 2) Use disposable drapes and covers exclusively in the contamination zone for easy cleaning. 3) Limit OR staff to 4 hours in the contamination zone, partially due to strict adherence to PPE requirements, especially N95 masks, which can be more uncomfortable than standard surgical masks. 4) Proactively rest staff every 4 hours to prevent dehydration when wearing heavy PPE; these staff are also isolated for a period of time before returning to new surgeries, depending on staff availability.

\section{Postoperative Management}

Suspected cases are not returned to general wards after surgery. Instead, these patients remain in special isolation wards within the designated contamination zone until test results come back negative.

Our approach to providing emergency care during the COVID-19 pandemic is designed to both prevent nosocomial spread of infection and efficiently treat the most urgent neurosurgical problems that must necessarily be dealt with even during this time. Using a principle of isolation of unknown cases and maximal PPE protection until COVID-19 status is known to be negative, we have had a record of zero infections among our team members and other patients at our referral center (the reported infection was at another hospital in Tianjin).

\section{General Recommendations}

Hospital staff attrition caused by infection can be extremely discouraging and devastating among the provider team and society as a whole. This happened to the healthcare providers at Wuhan, Hubei Province, in January 2020, due to limited knowledge and protection. As of March 30, there have been 2974 confirmed cases and more than 60 deaths among the local healthcare team in Hubei. The cases are centralized in major hospitals, and most of them are not from infectious or respiratory disease services. ${ }^{1}$ However, the situation improved dramatically after taking strict precautions - there is no infection reported among fully equipped healthcare workers who were recruited to support Wuhan province (6200 nationally, 628 from Tianjin) since February 2020. The standard precautions for the general public are absolutely inadequate for health workers. Don't reinvent the wheel. Please maximize protection in all ways-this is the firsthand experience from China to preserve healthcare resources for the battle against this epidemic.

https://thejns.org/doi/abs/10.3171/2020.4.JNS201018

\section{References}

1. Nanfang Weekend News. Article in Chinese. April 3, 2020. Accessed April 8, 2020. https://www.infzm.com/ contents $/ 180770$

\section{Disclosures}

The authors report no conflict of interest.

\section{Correspondence}

Xiaoguang Tong: tongxg@yahoo.com.

INCLUDE WHEN CITING

Published online April 24, 2020; DOI: 10.3171/2020.4.JNS201018. 